\title{
PARP3, a new therapeutic target to alter Rictor/mTORC2 signaling and tumor progression in BRCA1-associated cancers
}

\author{
Carole Beck ${ }^{1}$ - José Manuel Rodriguez-Vargas ${ }^{1}$ - Christian Boehler ${ }^{1}$ Isabelle Robert ${ }^{2,3,4,5}$ - Vincent Heyer ${ }^{2,3,4,5}$. \\ Najat Hanini ${ }^{1} \cdot$ Laurent R. Gauthier $^{6}$. Agnès Tissier ${ }^{7}$ - Valérie Schreiber ${ }^{1} \cdot$ Mikael Elofsson $^{8}$. \\ Bernardo Reina San Martin ${ }^{2,3,4,5} \cdot$ Françoise Dantzer $^{1}$
}

Received: 16 March 2018 / Revised: 7 September 2018 / Accepted: 22 October 2018 / Published online: 15 November 2018

(c) ADMC Associazione Differenziamento e Morte Cellulare 2018

\begin{abstract}
PARP3 has been shown to be a key driver of TGF $\beta$-induced epithelial-to-mesenchymal transition (EMT) and stemness in breast cancer cells, emerging as an attractive therapeutic target. Nevertheless, the therapeutic value of PARP3 inhibition has not yet been assessed. Here we investigated the impact of the absence of PARP3 or its inhibition on the tumorigenicity of BRCA1-proficient versus BRCA1-deficient breast cancer cell lines, focusing on the triple-negative breast cancer subtype (TNBC). We show that PARP3 knockdown exacerbates centrosome amplification and genome instability and reduces

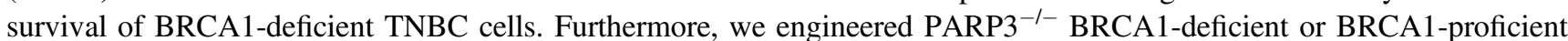
TNBC cell lines using the CRISPR $/ \mathrm{nCas}^{\mathrm{D} 10 \mathrm{~A}}$ gene editing technology and demonstrate that the absence of PARP3 selectively suppresses the growth, survival and in vivo tumorigenicity of BRCA1-deficient TNBC cells, mechanistically via effects associated with an altered Rictor/mTORC2 signaling complex resulting from enhanced ubiquitination of Rictor. Accordingly, PARP3 interacts with and ADP-ribosylates GSK3 $\beta$, a positive regulator of Rictor ubiquitination and degradation. Importantly, these phenotypes were rescued by re-expression of a wild-type PARP3 but not by a catalytic mutant, demonstrating the importance of PARP3's catalytic activity. Accordingly, reduced survival and compromised Rictor/mTORC2 signaling were also observed using a cell-permeable PARP3-specific inhibitor. We conclude that PARP3 and BRCA1 are synthetic lethal and that targeting PARP3's catalytic activity is a promising therapeutic strategy for BRCA1-associated cancers via the Rictor/mTORC2 signaling pathway.
\end{abstract}

These authors contributed equally: Carole Beck, José Manuel Rodriguez-Vargas

Edited by: J.P. Medema

Electronic supplementary material The online version of this article (https://doi.org/10.1038/s41418-018-0233-1) contains supplementary material, which is available to authorized users.

$\triangle$ Françoise Dantzer

francoise.dantzer@unistra.fr

1 Poly(ADP-ribosyl)ation and Genome Integrity, Laboratoire d'Excellence Medalis, UMR7242, Centre Nationale de la Recherche Scientifique/Université de Strasbourg, Institut de Recherche de l'Ecole de Biotechnologie de Strasbourg, 300 bld. S. Brant, CS10413, 67412 Illkirch, France

2 Institut de Génétique et de Biologie Moléculaire et Cellulaire (IGBMC), Illkirch, France

3 Institut National de la Santé et de la Recherche Médicale (INSERM), U964 Illkirch, France

\section{Introduction}

Breast cancer susceptibility gene 1 (BRCA1) is a wellknown tumor suppressor and women carrying a germline mutation in the gene have a significantly higher risk to develop breast and ovarian cancer. Poly(ADP-ribose) polymerase (PARP) inhibition by targeting PARP1 is

4 Centre National de la Recherche Scientifique (CNRS), UMR7104 Illkirch, France

5 Université de Strasbourg, Illkirch, France

6 Laboratoire de radiopathologie, CEA-DRF/INSERM U967, Institut de biologie François Jacob, Institut de Radiobiologie Cellulaire et Moléculaire (IRCM), 18 route du Panorama, 92265 Fontenay-aux-Roses, France

7 EMT and Cancer Cell Plasticity, Centre de Recherche en Cancérologie, UMR INSERM 1052 CNRS 5286, Centre Léon Bérard, Lyon F-69008, France

8 Department of chemistry, Umea University, Umea, Sweden 
nowadays considered as one of the most pervasive therapeutic opportunity to sensitize BRCA1-deficient tumors in a synthetic lethality approach, owing to its prominent role in the maintenance of genome integrity. This attractive therapeutic potential has encouraged pharmaceutical companies to design potent PARP inhibitors resulting in several leading candidates that have entered clinical trials. However, the PARP family consists of 17 members having distinct structural features and non-redundant biological functions, but sharing a highly conserved PARP catalytic domain [1]. Thus it is still unclear how many different PARP members are targeted by the currently used PARP inhibitors [2]. Therefore, it appears fundamental to determine the biological properties of the less-characterized PARPs and explore the therapeutic benefit of their inhibition in cancer therapy.

Frequently the most aggressive BRCAl mutationassociated tumors lack expression of estrogen receptor, progesterone receptor and HER2 receptor being classified as triple-negative breast cancers (TNBC) [3]. These tumors represent a difficult therapeutic challenge owing to their cell heterogeneity, the lack of validated molecular targets and the poor outcome of the patients. Thus, achieving a better understanding of the signaling pathways driving TNBC is determinant to identify novel therapeutic targets and develop new curative strategies. It has been shown that basal-like TNBC cells exploit the Rictor/mTORC2 signaling pathway to promote tumor progression [4].

mTORC2 together with mTORC1 represent two structurally distinct multiprotein complexes of the mammalian target of rapamycin (mTOR), a serine/threonine kinase influencing cell metabolism, proliferation, survival, and tumor growth [5]. mTORC1 consists of mTOR, Raptor, mLST8, and PRAS40 and is well characterized for it role in protein and lipid synthesis, mitochondrial metabolism and autophagy. mTORC2 comprises mTOR, mLST8, Rictor, mSIN1, and Protor and functions as a critical Serine 473 kinase of Akt, often hyper-activated in cancers [6]. Rictor/ mTORC2 mediates cell survival, chemoresistance, cytoskeleton reorganization, cell motility, and TGF $\beta$-induced epithelial-to-mesenchymal transition (EMT), key hallmarks of the metastatic process. In this complex, Rictor is defined as an essential scaffold protein required for mTORC2 assembly, stability, and function [7].

An advanced analysis of the published PARP3 expression profile in the panel of breast cancer cells from the Cancer Cell Line Encyclopedia (CCLE) revealed a significantly higher expression of $P A R P 3$ in the basal-like TNBC subtypes compared to the non-TNBC (Supplementary Fig. 1). Initially, the DNA-dependent PARP3 was described to play critical roles in the repair of double-strand breaks via non-homologous end joining (NHEJ), in class switch recombination, in chromosomal rearrangements by suppressing G4 structures, in telomere segregation and microtubule spindle formation during mitosis and in transcriptional regulation during development in the zebrafish [8-13]. Recently, PARP3 emerged as a promising therapeutic target to restrain TGF $\beta$ and ROS-driven EMT and limit stemness in breast cancer cells [14]. However, the beneficial significance of PARP3 inhibition to prevent tumor progression has not yet been evaluated.

Here we examined the impact of the absence of PARP3 and its chemical inhibition on the tumorigenicity of BRCA1-proficient versus BRCA1-deficient TNBC cell lines. We demonstrate that PARP3 inactivation selectively suppresses the tumor progression of BRCA1-deficient TNBC cells via effects associated with impaired Rictor/ mTORC2 signaling, defective cytoskeleton organization and exacerbated centrosomal amplification. This study supports PARP3 inhibition as an encouraging targeted therapy option for BRCA1-deficient TNBC.

\section{Material and methods}

\section{Reagents}

TGF $\beta 2$ and MG132 were purchased from Sigma-Aldrich. The PARP1 inhibitor Ku-0058948 and the PARP3 inhibitor ME0328 have been described [15-17]. The PARG inhibitor PDD 00017273 was purchased from Tocris Bioscience (Bristol, UK).

\section{Cell lines and cell culture}

MDA-MB231, Hs578T, and MDA-MB436 (ATCC) are defined as basal-like TNBC cells [18]. MDA-MB436 cell line harbors a $5396+1 \mathrm{G}>\mathrm{A}$ BRCA1 mutation in the splice donor site of exon 20. MDA-MB231 and MDA-MB436 cells were grown in RPMI supplemented with $10 \%$ fetal calf serum and $1 \%$ gentamicin. Hs578T were grown in DMEM-1g/L D-glucose supplemented with $20 \%$ fetal calf serum and $1 \%$ gentamicin. All cell lines were maintained at $37{ }^{\circ} \mathrm{C}$ and $5 \% \quad \mathrm{CO}_{2}$. Flag, Flag-PARP $3^{\mathrm{WT}}$ and Flag$\mathrm{PARP}^{\mathrm{HE}}$ rescued $\mathrm{PARP}^{-l-\mathrm{c}}$ MDA-MB436 cell lines were maintained in $0.2 \mu \mathrm{g} / \mathrm{mL}$ Puromycin-containing medium. When indicated, cells were treated with $10 \mathrm{ng} / \mathrm{mL}$ of TGF $\beta 2$ for $48 \mathrm{~h}$ before processing.

\section{siRNA-mediated depletion}

Gene-specific siRNAs (ON_TARGET plus smart pool) for PARP3 (L-009297), PTEN (J-003023), BRCA1 (J003461), and the negative control siRNA (D-001810) were obtained from Dharmacon (Thermo Fisher Scientific). Cells were transfected with $50 \mathrm{nM}$ siRNA using JetPrime 
(PolyPlus transfection) according to the manufacturer's instructions and cells were processed for the indicated experiments from $48 \mathrm{~h}$ to $72 \mathrm{~h}$ later.

\section{Knockout of PARP3 using CRISPR/nCas9-mediated genome editing}

Cells were co-transfected with two plasmids expressing 2 gRNAs targeting exon 2 and co-expressing nCas9-EGFP and 2 gRNAs targeting exon 5 and co-expressing nCas9mCherry and bearing Neomycin or Hygromycin selection cassettes respectively (Sup. Figure 4). Forty-eight hours after transfection, EGFP + mCherry + cells were sorted by flow cytometry and cultured for 3 days in medium containing Hygromycin $(350 \mu \mathrm{g} / \mathrm{mL})$ and $\mathrm{G} 418(500 \mu \mathrm{g} / \mathrm{mL})$ for MDA-MB231 or Hygromycin $(400 \mu \mathrm{g} / \mathrm{mL})$ and $\mathrm{G} 418$ $(350 \mu \mathrm{g} / \mathrm{mL})$ for MDA-MB436. Single colonies were picked, amplified, and genotyped by PCR using primers located upstream of exon 2 and downstream of exon 5 (Suplementary Table S1). PCR products were sequenced and the absence of PARP3 was verified by western blot.

\section{gRNA sequences}

The gRNA sequences are as following: gRNA1 (GCCTC AGCGGTGGAGCGGAA, Exon 2), gRNA2 (AGAGAAG CGCATAATCCGCG, Exon 2), gRNA3 (GTTAGTGAT GAGCTTCTGCG, Exon 5), gRNA4 (CACCATGGCCC TCATGGACC, Exon 5) (Supplementary Fig. 4A). nCas9compatible gRNA pairs were selected using the CRISPR design tool (http://crispr.mit.edu/).

\section{Generation of PARP3-rescued cell lines}

$P A R P 3^{-l-c}$ MDA-MB436 cells were transfected with $10 \mu \mathrm{g}$ of plasmids encoding Flag, Flag-PARP3 ${ }^{\text {WT }}$ or Flag$\mathrm{PARP}^{\mathrm{HE}}$ using JetPrime. Two days after transfection, cells were selected for 2 days with Puromycin $(0.8 \mu \mathrm{g} / \mathrm{mL})$, maintained for 2 weeks under Puromycin $(0.2 \mu \mathrm{g} / \mathrm{mL})$ and sorted for EGFP expression by flow cytometry. Expression was verified by western blot.

\section{Cell extracts and western blot}

Cells were lysed by incubation on ice for $30 \mathrm{~min}$ in RIPAlike buffer $(50 \mathrm{mM}$ Tris-HCl, $\mathrm{pH} 8,1 \%$ Triton X-100, $0.25 \% \mathrm{Na}$ Deoxycholate, $150 \mathrm{mM} \mathrm{NaCl}, 1 \mathrm{mM}$ EDTA, 50 $\mathrm{mM} \mathrm{NaF}, 20 \mathrm{mM}$ Na pyrophosphate, $1 \mathrm{mM} \mathrm{Na}$ orthovanadate, $1 \mathrm{mM}$ Pefabloc (Roche), $1 \mathrm{X}$ protease inhibitor cocktail (Roche)). After centrifugation at $13,000 \mathrm{rpm}$ at $4{ }^{\circ} \mathrm{C}$ for $15 \mathrm{~min}$, cleared suspension was quantified by Bradford protein assay. Proteins were analysed by SDS-PAGE and immunoblotting using the appropriate antibodies
(Supplementary Table S2). When analysing the impact of the ME0328, cells were treated with the compound for $24 \mathrm{~h}$ before lysis.

\section{Immunoprecipitation experiments}

Equivalent amounts of RIPA-like cell extracts (1.5-2 mg total proteins) were diluted in dilution buffer DB $(20 \mathrm{mM}$ Tris- $\mathrm{HCl}, \mathrm{pH} 7.5,0.1 \%$ NP40, $150 \mathrm{mM} \mathrm{NaCl}, 1 \mathrm{mM}$ Pefabloc) and pre-cleared by incubation on protein $\mathrm{A} / \mathrm{G}$ separose beads for $1 \mathrm{~h}$ at $4{ }^{\circ} \mathrm{C}$ before incubation with the indicated antibodies (Supplementary Table S2) overnight at $4{ }^{\circ} \mathrm{C}$ followed by $2 \mathrm{~h}$ incubation at $4{ }^{\circ} \mathrm{C}$ with protein $\mathrm{A} / \mathrm{G}$ sepharose (GE Healthcare, Little Chalfont, UK). Beads were washed twice with $\mathrm{DB}$ containing $250 \mathrm{mM} \mathrm{NaCl}$ and twice with DB containing $150 \mathrm{mM} \mathrm{NaCl}$. Beads were then resuspended in Laemmli buffer and analysed by SDSPAGE and immunoblotting as above.

When analyzing the ADP-ribosylation of GSK3 $\beta$, cells were pre-treated with the PARG inhibitor PDD 00017273 at $1 \mu \mathrm{M}$ for $2 \mathrm{~h}$ to prevent from poly(ADP-ribose) degradation and the compound was maintained throughout. The PARP inhibitor $\mathrm{Ku}-0058948$ was added during lysis at $100 \mathrm{nM}$ to prevent from unspecific ADP-ribosylation during lysis.

\section{In vivo ubiquitination assay}

Cells were transfected with $5 \mu \mathrm{g}$ of HA-Ubiquitin [19] for $48 \mathrm{~h}$ and either mock-treated or treated with MG132 (10 $\mu \mathrm{M})$ for $12 \mathrm{~h}$ before lysis using the RIPA-like buffer. Equivalent amounts of total protein extracts $(2 \mathrm{mg})$ were processed for immunoprecipitation as above using the antiRictor antibody (Supplementary Table S2). Ubiquitinated Rictor was revealed by western blotting using an anti-HA antibody (Supplementary Table S2).

\section{Colony-forming assay and cell proliferation}

siRNA-depleted cells were collected $72 \mathrm{~h}$ after siRNA transfection. Cells were seeded in triplicates at 1000 cells for MDA-MB231, 1500 cells for MDA-MB436 and 1500 cells for HS578T in 100-mm culture dishes. PARP3 ${ }^{-1-}$ MDA-MB231 clones were seeded in triplicates at the number of 1000 cells and PARP3 ${ }^{-1-}$ MDA-MB436 clones were seeded at the number of 3000 cells in $100-\mathrm{mm}$ culture dishes. When analysing the impact of ME0328, cells were pre-incubated with the compound for $24 \mathrm{~h}$ before seeding and the medium with the compound was renewed every 48 h. From 7 to 15 days later, cells were fixed for $30 \mathrm{~min}$ in formaldehyde (3.7\%), stained with crystal violet $(0.1 \%)$ and colonies were scored. Statistical analyses were determined by ANOVA tests as indicated by $p$-values using StatView software. To determine cell growth rate, cells were seeded 
into 6-well plates (40,000 cells/well for MDA-MB231 cells and 80,000 cells/well for MDA-MB436 cells) in triplicate and counted daily for 4 days.

\section{Immunofluorescence microscopy and fluorescent in situ hybridization (FISH)}

F-actin labeling was performed using Alexa Fluor 568 Phalloidin (Thermo Fisher Scientific) according to the manufacturer's protocol. For immunofluorescence, cells were fixed in $4 \%$ formaldehyde and stained with the indicated antibodies as described [8]. Metaphase spreads and analysis of telomere aberrations by FISH were performed as described [8].

\section{GTPase activity assays}

Cells were serum-starved for $24 \mathrm{~h}$ and stimulated with TGF 32 (10 ng/mL) for $5 \mathrm{~h}$. GTP-bound RhoA and Rac1 and total protein contents were detected using Active Rho (16116) and Active Rac1 (16118) pull-down and detection kits (Thermo Fisher Scientific) according to manufacturer's instructions.

\section{Scratch wound assays}

A linear wound was created on confluent monolayers of cells using a sterile $200 \mu \mathrm{l}$ pipette tip. Cells were placed in an environment controlled wide field microscope Leica DMIRE 2 microscope equipped with a Photometrics Prime sCMOS camera and the Imaging capture software Metamorph, and imaged every $20 \mathrm{~min}$ for $24 \mathrm{~h}$ using a $\times 10$ phase contrast objective.

\section{In vivo tumorigenicity experiments}

Animal protocols were approved by the Ministry of Higher Education in Research and Innovation and the local ethics committee Cremeas. Female athymic nude mice (S/SOPF SWISS NU/NU) were purchased from Charles Rivers Laboratories. For xenograft studies, $3.5 \times 10^{6}$ MDA-MB231 and $5 \times 10^{6}$ MDA-MB436 cells in $50 \%$ of Matrigel (Corning) were implanted subcutaneously into both flanks of the 7 -week-old nude mice upon xylazine $(50 \mathrm{mg} / \mathrm{kg}) /$ ketamine $(3 \mathrm{mg} / \mathrm{kg})$ anesthesia. Tumor volumes were calculated from caliper measurements by length (L) and width (W) by using the formula: Tumor volume $\left(\mathrm{V} \mathrm{mm}^{3}\right)=$ length $\times(\text { Width })^{2} / 2$.

\section{In vitro PARylation assays}

In vitro PARylation assays were performed using immunopurified Flag-PARP $3^{\mathrm{WT}}$ and Flag-PARP ${ }^{\mathrm{HE}}$ in activity buffer containing $\alpha-{ }^{32}$ PNAD and DNase I activated calf thymus DNA as described [8].

\section{Results}

\section{PARP3 silencing impairs survival of BRCA1-deficient or BRCA1-depleted TNBC cells}

Tankyrase 1 inhibition was found to be selectively lethal in the context of BRCA1 deficiency [20]. Therefore, we investigated the possibility that targeting PARP3, an activator of Tankyrase 1 [8], may have a similar effect. We used breast cancer cells of the basal-like TNBC subtypes because of the predominant expression of PARP3 (Supplementary Fig. 1) [14]. We first compared the sensitivity of the BRCA1-proficient (MDA-MB231, Hs578T) and BRCA1-deficient (MDA-MB436) cells to the depletion of PARP3 by clonogenic survival assays. PARP3 knockdown was significantly more lethal in the BRCA1deficient cells (Fig. 1a). Consistent with this, knockdown of both PARP3 and BRCA1 in the BRCA1-proficient cells (MDA-MB231 and Hs578T) resulted in significantly reduced cell survival when compared to cells transfected with control siRNA or with each siRNA alone (Fig. 1b, c). A similar lethal impact of PARP3 silencing was observed in the BRCA1-deficient breast cancer cell line HCC1937 and in the ovarian cancer cell line UWB1.289, when compared to the isogenic cell lines with restored BRCA1 expression (Supplementary Fig. 3 and 7A). We conclude that PARP3 depletion is lethal in a BRCA1-deficient background.

\section{PARP3 silencing exacerbates centrosome amplification and genome instability in BRCA1- deficient TNBC cells}

Increased lethality associated with the combined deficiency of BRCA1 and Tankyrase 1 was previously specified by enhanced centrosome amplification [20]. PARP3 long isoform localizes to the daughter centriole and regulates mitotis $[8,21]$. Therefore, we interrogated on the consequence of PARP3 depletion on centrosome amplification in the BRCA1-proficient Hs578T cells versus the BRCA1-deficient MDA-MB436 cells. Although PARP3 depletion moderately increased the percentage of cells with centrosome amplification in the Hs578T cells (Fig. 2a, b), this phenotype was markedly exacerbated in the MDA-MB436 cells (Fig. 2a-c). Centrosome amplification often results in the formation of multiple nuclei within a single cell. Accordingly, the depletion of PARP3 intensified the percentage of cells with multiple nuclei in the MDA-MB436 (19.2\%) versus the Hs578T cells 


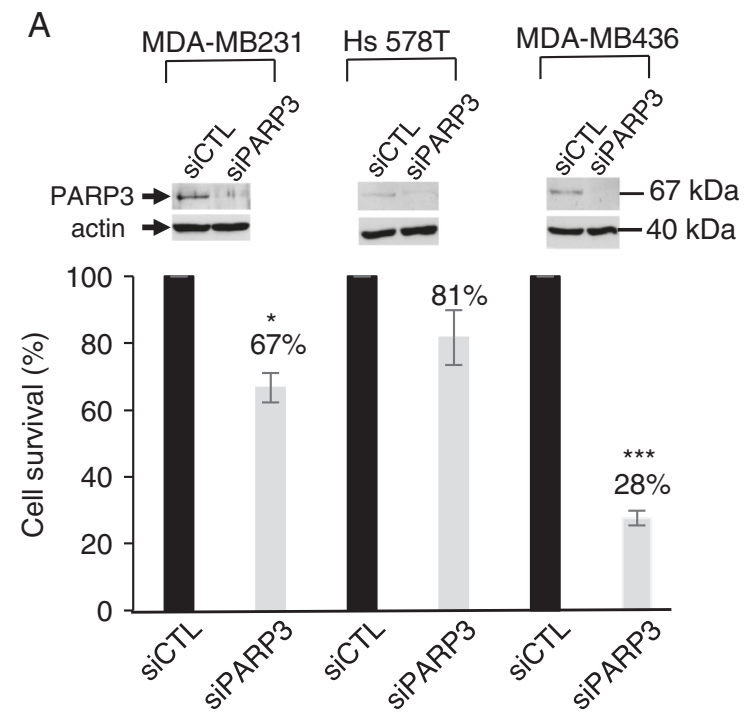

B

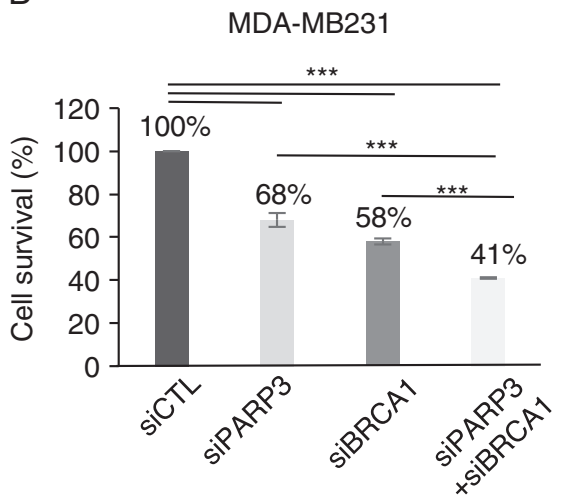

Fig. 1 PARP3 silencing is more lethal in the context of BRCA1 deficiency. a Clonogenic survival of MDA-MB231 and Hs578T breast cancer cells expressing a wild-type BRCA1 compared to the BRCA1deficient breast cancer cell line MDA-MB436 after transfection with the indicated siRNA. The survival of the si-control (siCTL) cells was set as $100 \%$. Experiments were performed more than three times giving similar results. Mean values of triplicates \pm SD are indicated. $* p<0.05 ; * * * p<0.001$. (Insets) The efficiency of the depletions for the experiments shown was verified by western blot analysis $72 \mathrm{~h}$ post

(12.7\%) (Fig. 2d). As centrosome amplification has been linked with genome instability in breast cancer [22], we scored telomeric aberrations and the emergence of micronuclei as indicators of overall chromosome instability. Although the depletion of PARP3 did not aggravate the telomere instability detected in the MDAMB436 cells, it significantly increased the formation of micronuclei in the MDA-MB436 cells only (Fig. 2e, f). Together, these findings revealed that the depletion of PARP3 results in supernumerary centrosomes and increased genome instability markedly in BRCA1deficient cells.

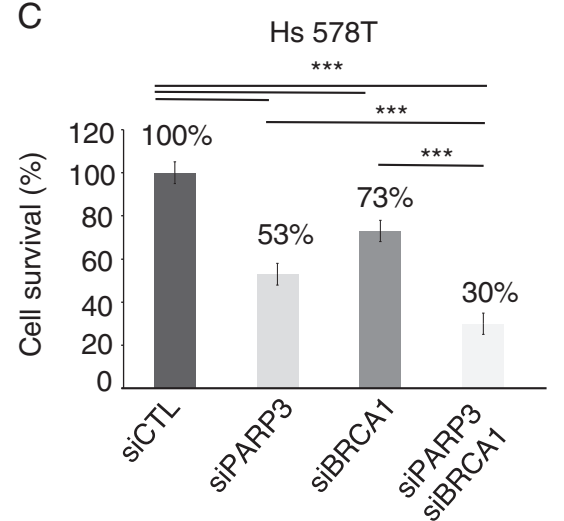

siRNA transfection. b, c Clonogenic survival of MDA-MB231 and Hs578T cells after transfection with the indicated siRNA. Note the additive effect of the co-depletion of both PARP3 and BRCA1 compared to the relative single depletions. The survival of the si-control (siCTL) cells was set as $100 \%$. Experiments were performed more than three times giving similar results. Mean values of triplicates \pm /SD are indicated. $* * * p<0.001$. The efficiency of depletions was verified by RT-qPCR (Supplementary Fig. 2)

\section{Knockout of PARP3 impairs proliferation, survival, and tumor progression in BRCA1-deficient TNBC cells}

To further clarify the function of PARP3 in breast cancer associated with BRCA1 deficiency, we inactivated PARP3 in MDA-MB231 and MDA-MB436 cells using a double nCas9(D10A) strategy (Supplementary Fig. 4 and [23]). For each cell line, three independent $\mathrm{PARP} 3^{-1-}$ clones were generated (Supplementary Fig. 5, Fig. 3a, b). The absence of PARP3 only moderately impaired the proliferation rate of the MDA-MB231 cells, whereas reduced markedly the proliferation rate of the MDA-MB436 cells (Fig. 3c, d). In 
A

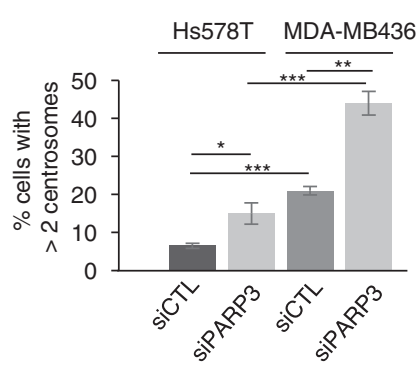

B

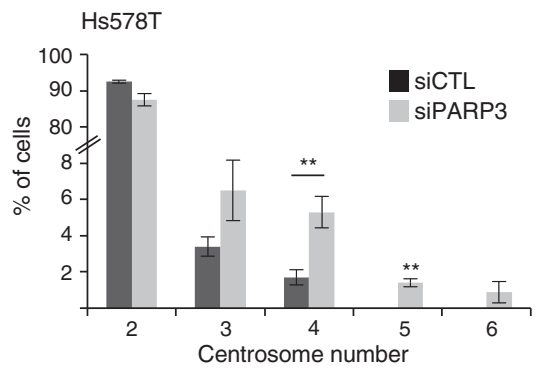

C

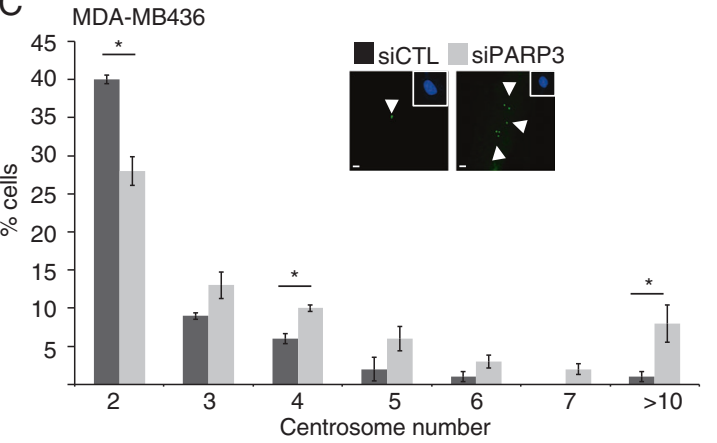

D
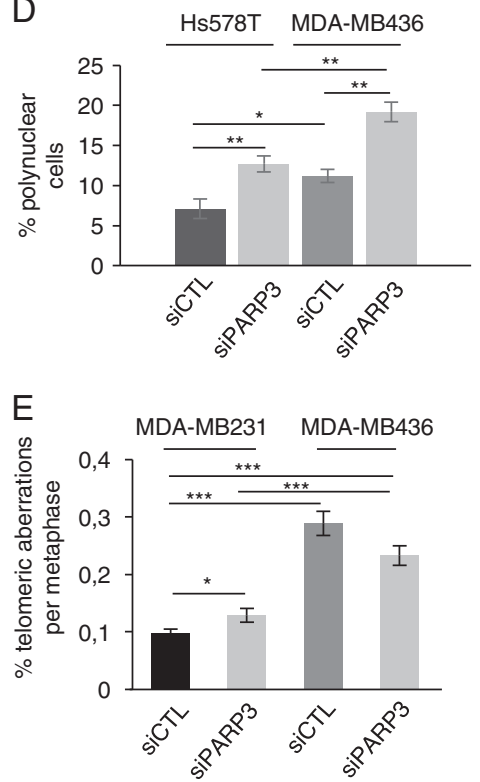

$\mathrm{F}$

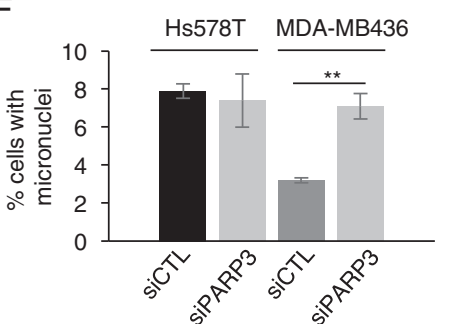

Fig. 2 PARP3 silencing exacerbates centrosome amplification and the appearance of polynuclear cells and induces genome instability in BRCA1-deficient cells. a Quantification of the percentage of total cells displaying more than two centrioles in Hs578T compared to MDAMB436 cells after transfection with the indicated siRNA. Experiments were performed three times. Mean values of the triplicates \pm SD are indicated. ${ }^{*} p<0.05 ;{ }^{*} p<0.01 ; * * * p<0.001$. b, c Detailed distribution of the percentage of cells displaying from 3 to $>10$ centrioles in Hs578T and MDA-MB436 cells. Whereas Hs578T cells displayed up to 6 centrosomes/cells upon PARP3 silencing, this number raised to over 10 centrosomes in $8 \%$ of the MDA-MB436 cells. An average of 500 cells per cell line was scored in more than 20 randomly selected fields. Results are averages from three independent experiments. Mean values $\pm \mathrm{SD}$ are indicated. $* p<0.05 ; * * p<0.01$. A representative image of the centrosome amplification observed in a PARP3-silenced MDA-MB436 cell (siPARP3) versus a control (siCTL) cell is shown. Scale bars $=1 \mu \mathrm{m}$. Centrosomes are stained with p34cdc2 (green) and cells are counterstained with DAPI (blue). d Quantification of the percentage of cells with multiple nuclei in Hs578T and MDA-MB436 cells transfected with the indicated siRNA. Cells with two or more nuclei were scored. An average of 500 cells per cell line was scored in more than 20 randomly selected fields. Results are averages from three independent experiments. Mean values $\pm \mathrm{SD}$ are indicated. ${ }^{*} p<0.05$; $* * p<0.01$. e Quantification of telomeric aberrations expressed as percentages of telomere aberrations per metaphase in MDA-MB231 and MDA-MB436 cells transfected with the indicated siRNA. Telomere aberrations were detected by FISH on metaphase spreads. Thirty cells were scored for each independent condition. Mean values \pm SD are indicated. $* p<0.05 ; * * * p<0.001$. f Quantification of the percentage of cells with micronuclei in Hs578T and MDA-MB436 cells transfected with the indicated siRNA. An average of 500 cells per cell line was scored in more than 20 randomly selected fields. Results are averages from three independent experiments. Mean values \pm SD are indicated. $* * p<0.01$

We then analysed the impact of PARP3 knockout on the in vivo tumorigenicity of the MDA-MB231 and MDAMB436 cells subcutaneously xenografted into nude mice. Whereas PARP3 knockout MDA-MB231 cells exhibited notably reduced tumorigenic potential with a higher effect in the PARP $3^{-1-2}$ clone compared to the parental MDAMB231 cells (Fig. 3g), the tumorigenic potential was totally 
A

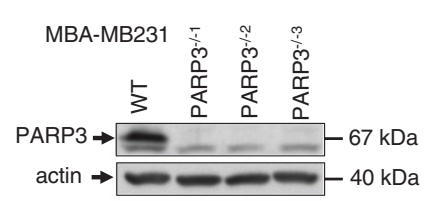

C

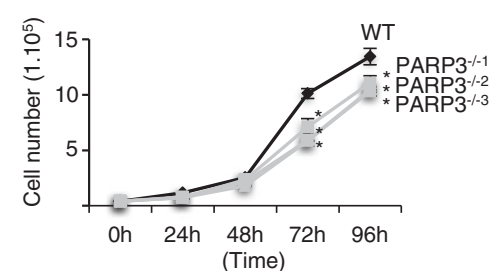

$\mathrm{E}$
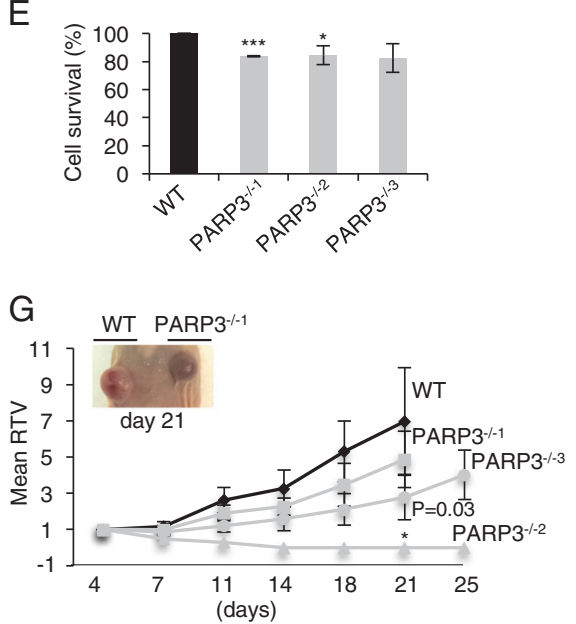

Fig. 3 Knockout of PARP3 significantly reduces cell growth and survival and suppresses tumor growth in the context of BRCA1 deficiency. a, b Western blot analysis of PARP3 expression in the wild type (WT) and three PARP $3^{-1-1}, \mathrm{PARP}^{-1-2}, \mathrm{PARP}^{-1-3} \mathrm{MDA}-$ MB231 clones as well as in the wild type (WT) and three PARP3 ${ }^{-l-a}$, $\mathrm{PARP}^{-l-\mathrm{b}}$, PARP $3^{-l-\mathrm{c}}$ MDA-MB436 clones selected upon screening and sequence analysis. Actin is used as loading control. The remaining lower band is unspecific and randomly detected by the PARP3 antibody depending on the cell lines used. c, d Graphs compare proliferation rates between wild-type MDA-MB231 (WT) and PARP3 ${ }^{-l-1}, \mathrm{PARP}^{-l-2}, \mathrm{PARP}^{-l-3}$ MDA-MB231 cell lines and between wild-type MDA-MB436 (WT) and PARP3 ${ }^{-l-\mathrm{a}}, \mathrm{PARP}^{-1-\mathrm{b}}, \mathrm{PARP} 3$

${ }^{-1-\mathrm{c}}$ MDA-MB436 clones. Experiments were performed three times giving similar results. Mean values of triplicates \pm SD are indicated. ${ }^{*} p<0.05 ;{ }^{*} p<0.01 ; * * * p<0.001$. e, f Survival is expressed as the percentage of colonies formed in the PARP $3^{-1-1}, \mathrm{PARP}^{-1-2}$, PARP3

abolished in the three $\mathrm{PARP}^{-1-\mathrm{a}}, \mathrm{PARP}^{-1-\mathrm{b}}, \mathrm{PARP}^{-1-\mathrm{c}}$ MDA-MB436 cells (Fig. 3h). In line with this, the silencing of PARP3 abolished the tumorigenic potential of the BRCA1-mutated HCC1937 cells and reciprocally the silencing of BRCA1 significantly reduced the tumorigenic potential of the PARP3 $^{-1-1}$ MDA-MB231 cells (Supplementary Fig. 6B and 7B).

Altogether, these data confirmed that the absence of PARP3 is selectively lethal with BRCA1 deficiency in three different TNBC cell lines.
B

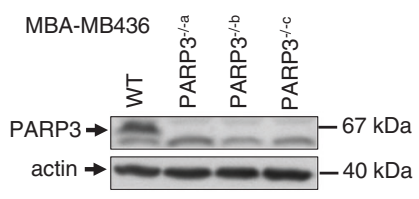

D

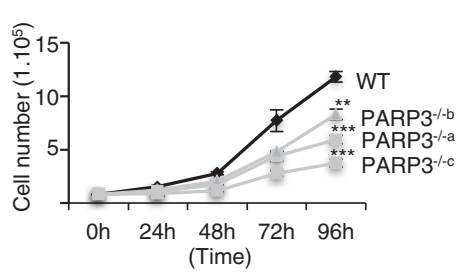

$\mathrm{F}$
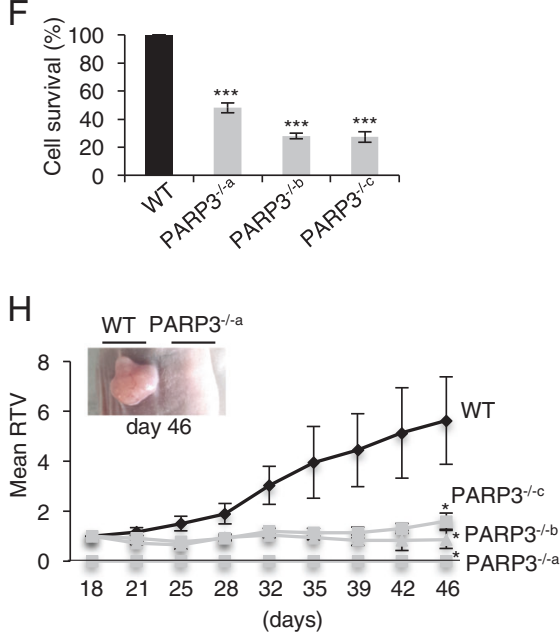

${ }^{-l-3}$ MDA-MB231 clones versus the parental MDA-MB231 (WT) cell line set to $100 \%$ and in the $\mathrm{PARP}^{-l-\mathrm{a}}$, $\mathrm{PARP}^{-I-\mathrm{b}}$ PARP3 $^{-I-\mathrm{c}}$ MDA-MB436 clones versus the wild-type MDA-MB436 (WT) set to $100 \%$. Results represent the mean values of three independent experiments \pm SD. ${ }^{*} p<0.05 ; * * * p<0.001$. g, h Relative tumor growth curves of xenografts derived from the wild-type (WT) MDA-MB231 cells or the three $\mathrm{PARP}^{-l-1}$ PARP $^{-l-2}$, PARP $^{-1-3}$ MDA-MB231 knockout cell lines and from the wild-type (WT) MDA-MB436 cells versus the PARP3 ${ }^{-l-\mathrm{a}}, \mathrm{PARP}^{-l-\mathrm{b}}$, PARP3 ${ }^{-1-\mathrm{c}}$ MDA-MB436 clones. Mean RTV (relative tumor volume) $(n=6$ individual mice) are expressed compared to tumor volumes on day 4 for MDA-MB231 series and on day 18 for the MDA-MB436 series. Insets, representative images of the tumors collected at the end of the experiment are shown. For $\mathbf{g}$, WT MDA-M231cells were implanted on the left flank, PARP $3^{-l-1}$ cells were implanted right. For $\mathbf{h}$, WT MDAMB436 cells were implanted left, $\mathrm{PARP}^{-l-\mathrm{a}}$ were implanted right

\section{Re-expression of PARP3, but not a catalytic mutant, rescues proliferation, survival and in vivo tumorigenicity in a BRCA1-deficient background}

To evaluate the functional importance of PARP3's catalytic activity in the context of BRCA1 deficiency and to exclude potential off-targets effects of the CRISPR/Cas9 system, we re-expressed Flag-PARP3 (Flag-PARP3 ${ }^{\mathrm{WT}}$ ), a catalytically inactive mutant (Flag-PARP3 ${ }^{\mathrm{HE}}$ ) or a Flag peptide alone in $\mathrm{PARP}^{-l-\mathrm{c}}$ MDA-MB436 cells. Flag-PARP3 ${ }^{\mathrm{HE}}$ was 
A
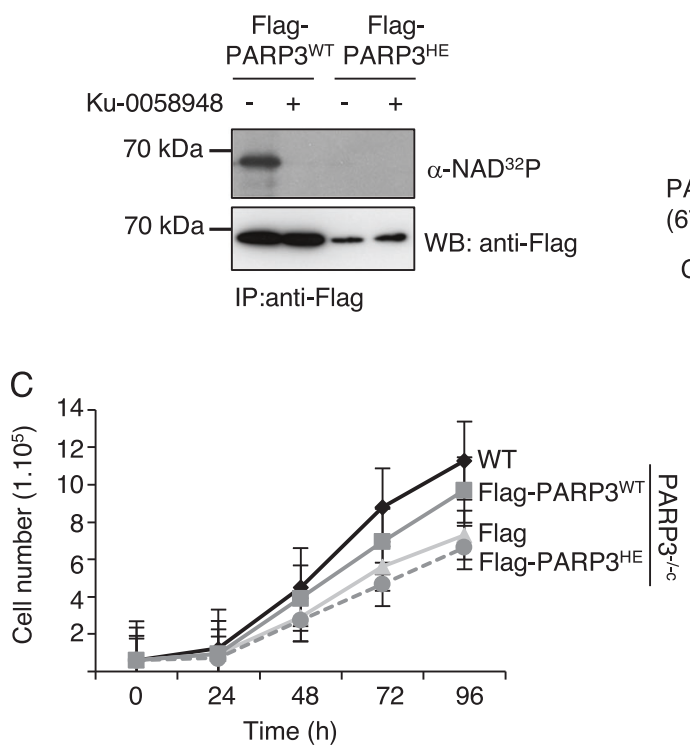

$E$
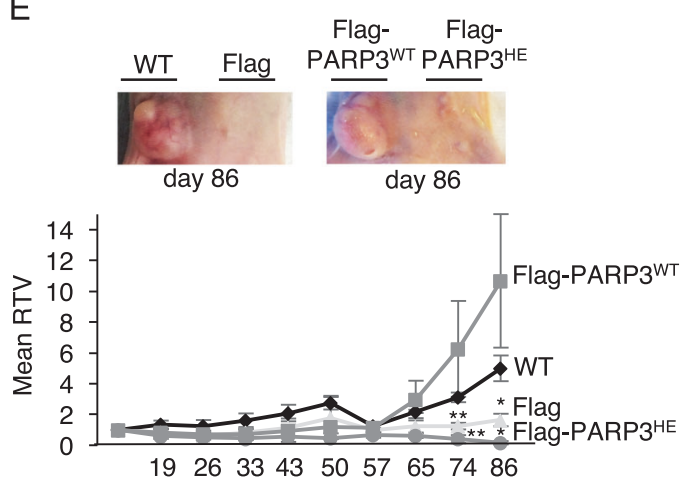

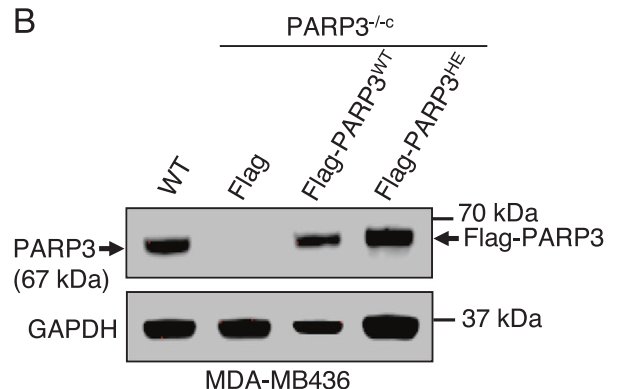

D
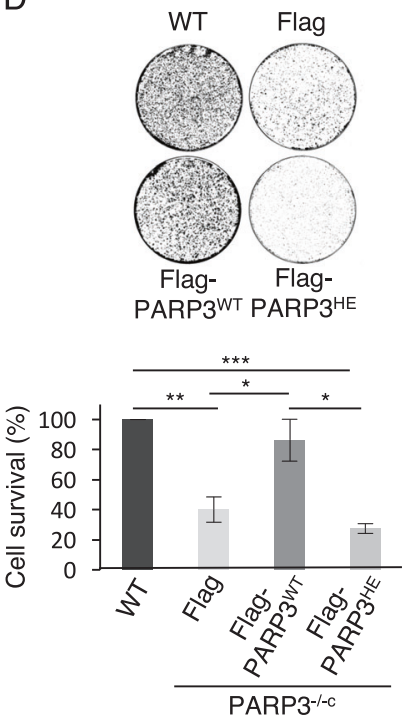

Fig. 4 Defective proliferation, survival, and in vivo tumorigenicity of the PARP3 knockout MDA-MB436 cells are rescued by the reexpression of the catalytically active PARP3 but not by the reexpression of a catalytically dead PARP3. a Radioactive in vitro autoADP-ribosylation assay showing the automodification of FlagPARP $3^{\text {WT }}$, although no automodification is detected for the dead Flag-PARP $3^{\mathrm{HE}}$ mutant. Immunopurified Flag-PARP $3^{\mathrm{WT}}$ and Flag$\mathrm{PARP}^{\mathrm{HE}}$ were incubated in PARP activity buffer containing DNAse I-treated DNA and $\alpha-{ }^{32}$ P-NAD ${ }^{+}$. The addition of Ku-0058948 (250 $\mathrm{nM}$ ) inhibits PARP3 [8]. b Western blot analysis of PARP3 expression in the wild type (WT) and PARP3 ${ }^{-I-c}$ MDA-MB436 cells with a stable expression of either the Flag control (Flag), Flag-PARP3 ${ }^{\text {WT }}$ or Flag-PARP $3{ }^{\mathrm{HE}}$. GAPDH is used as loading control. c Graphs compare proliferation rates between the parental MDA-MB436 (WT) and the PARP $^{-l-\mathrm{c}}$ MDA-MB436 cell lines expressing either the Flag control (Flag), Flag-PARP3 ${ }^{\mathrm{WT}}$ or Flag-PARP3 ${ }^{\mathrm{HE}}$ fusion proteins. Experiments were performed three times giving similar results. Mean values of

generated by introducing mutations (H376A and E508A) in the ADP-ribosyl transferase (ART) domain of PARP3. The effectiveness of this substitution was verified using an in vitro auto-ADP-ribosylation assay (Fig. 4a) and both Flag-tagged proteins were efficiently expressed in the restored cell lines (Fig. 4b). As expected, PARP ${ }^{-1-\mathrm{c}}$ MDA-MB436 cells expressing the Flag tag alone displayed reduced growth rate triplicates \pm SD of a representative experiment are indicated. d Survival is expressed as the percentage of colonies formed in the PARP3 ${ }^{-l-\mathrm{c}}$ MDA-MB436 cell lines expressing either the Flag control (Flag), Flag-PARP $^{\text {WT }}$ or Flag-PARP $^{\text {HE }}{ }^{\text {versus the parental MDA-MB436 }}$ cells (WT) set to $100 \%$. Results represent the mean values of three independent experiments \pm SD. $* p<0.05 ; * * p<0.01 ; * * * p<0.001$. Insets, an illustration of a representative experiment is shown. e Relative tumor growth curves of xenografts derived from the wild type (WT) MDA-MB436 cells, and the PARP $3^{-l-\mathrm{c}}$ MDA-MB436 cell lines expressing either the Flag control (Flag), Flag-PARP $3^{\text {WT }}$ or Flag$\mathrm{PARP}^{\mathrm{HE}}$ fusion proteins. Mean $\mathrm{RTV} \pm \mathrm{SD}(n=6$ individual mice $)$ are expressed compared to tumor volumes on day 13 for all cell lines. $* p<0.05 ; * * p<0.01$. Insets, representative images of the tumors collected at the end of the experiments. Illustration left, WT cells were implanted on the left flank, PARP $3^{-l-c}$-Flag cells were implanted right. Illustration right, $\mathrm{PARP}^{-l-\mathrm{c}}$-Flag-PARP $3^{\mathrm{WT}}$ cells were implanted left, PARP $3^{-l-c}$-Flag-PARP $3^{\mathrm{HE}}$ were implanted right

compared to the parental cell line (WT). Whereas a partial rescue of the proliferation defect was observed in the PARP3 ${ }^{-l-\mathrm{c}}$ MDA-MB436 cells expressing Flag-PARP ${ }^{\text {WT }}{ }^{\mathrm{WT}}$, no rescue was observed with the Flag-PARP ${ }^{\mathrm{HE}}$ mutant (Fig. 4c). To validate these observations further, we analysed the impact on cell survival using clonogenic assays (Fig. 4d). In support of Fig. 3, PARP $3^{-l-\mathrm{c}}$ MDA-MB436 cells expressing the Flag tag 
only exhibited profound decrease in cell survival compared to the parental cell line. While a substantial rescue was obtained in the PARP3 ${ }^{-l-\mathrm{c}}$ MDA-MB436 cells expressing Flag$\mathrm{PARP}^{\mathrm{WT}}$, no survival was retrieved in the $\mathrm{PARP}^{-l-\mathrm{c}}$ MDA-MB436 cells expressing Flag-PARP ${ }^{\mathrm{HE}}$. Next, we analyzed the in vivo tumorigenic potential of the rescued cell lines (Fig. 4e). PARP $^{-1-\mathrm{c}}$ MDA-MB436 cells expressing the Flag tag displayed a significantly decreased tumorigenic potential when compared to the parental cell line. Similarly, re-expression of Flag-PARP $3^{\mathrm{HE}}$ had no effect on tumor growth. On the contrary, re-expression of Flag-PARP3 ${ }^{\text {WT }}$ exacerbated tumor growth. Together, these results demonstrate that the catalytic activity of PARP3 is required for tumor growth in a BRCA1-deficient background.

\section{Knockout of PARP3 attenuates the oncogenic Rictor/mTORC2 signaling in the BRCA1-deficient TNBC cells}

In cancer progression, proliferation, survival, and centrosome amplification are highly dependent on the activation status of the oncogenic Rictor/mTORC2 signaling pathway. mTORC2 mediates the phosphorylation of Akt on Ser473 and contributes to BRCA1-mediated tumorigenesis [24, 25].

To examine the role of PARP3 in the Rictor/mTORC2 pathway in the context of BRCA1 deficiency, we tested the basal phosphorylation status of Akt on Ser 473 (Fig. 5a). Consistent with published reports, BRCA1deficient MDA-MB436 showed constitutive levels of pAkt(S473) compared to undetectable levels in the MDAMB231. Knockout of PARP3 significantly reduced the amount of p-Akt(S473) in the MDA-MB436 suggesting that PARP3 promotes the phosphorylation of Akt. To verify this observation further, we analysed the autophosphorylation of mTORC2 on Ser 2481 defined as a marker for intact mTORC2 activity [26]. We detected increased levels of p-mTORC2(S2481) in the parental MDAMB436 cells compared to the MDA-MB231 cells but no visible impact was seen in the absence of PARP3. We then analysed the expression of Rictor, a mandatory cofactor of mTORC2 required for its stability and integrity [6]. Similarly to PARP3, RICTOR transcript levels are higher in TNBC versus non-TNBC cells (Supplementary Fig. 1). The absence of PARP3 did not affect the expression of Rictor in the MDA-MB231 cells, but induced a notable decrease of Rictor expression in the MDA-MB436 cells. In contrast, the absence of PARP3 did not affect the mTORC1-mediated phosphorylation of p70S6K on Thr 389 indicating functional mTORC1 activity [26].

Next, to evaluate the contribution of PARP3 activity, we analysed the status of p-Akt(S473) in the MDA-MB436 cells expressing either Flag control, Flag-PARP $3^{\mathrm{WT}}$ or FlagPARP $^{\mathrm{HE}}$ (Fig. 5b). Consistent with the above results, PARP $^{-l-c}$ MDA-MB436 cells expressing the Flag tag only exhibited strong decrease in the basal level of p-Akt (S473) compared to the WT MDA-MB436 cells. Although the exogenous expression of Flag-PARP ${ }^{\text {WT }}$ rescued p-Akt (S473) levels, no rescue was detected upon re-expression of the dead Flag-PARP ${ }^{\mathrm{HE}}$ mutant. Similarly, we detected the reduced expression of Rictor in the $\mathrm{PARP}^{-1-\mathrm{c}}$ MDAMB436 cells expressing the Flag tag only compared to the WT MDA-MB436 cells. Whereas the re-expression of FlagPARP ${ }^{\mathrm{WT}}$ restored Rictor expression to a WT level, no rescued expression was seen in the $\mathrm{PARP}^{-1-\mathrm{c}}$ MDAMB436 complemented with Flag-PARP3 ${ }^{\mathrm{HE}}$. We also analysed the phosphorylation status of GSK3 $\beta$ on Ser 9, a target of activated Akt [27]. We observed reduced p-GSK3 $\beta$ (S9) levels in the PARP ${ }^{-1-\mathrm{c}}$ MDA-MB436 cells expressing the Flag tag only compared to the WT cells. Restored p-GSK3B(S9) levels were seen in the PARP ${ }^{-1-\mathrm{c}}$ MDAMB436 cells expressing Flag-PARP3 ${ }^{\text {WT }}$ but not in the $\mathrm{PARP}^{-1-\mathrm{c}}$ MDA-MB436 cells expressing Flag-PARP ${ }^{\mathrm{HE}}$. In support of these data, we confirmed reduced Rictor, pAkt(S473) and p-GSK3ß(S9) levels in the HCC1937 cells upon PARP3 silencing and in the two PARP3 knockout MDA-MB231 clones upon BRCA1 silencing while PTEN silencing had no impact (Supplementary Fig. 6C and 7C). We conclude that in BRCA1-deficient cells specifically, PARP3 activity promotes Rictor/mTORC2 signaling and its inhibition efficiently represses this tumorigenic pathway.

\section{PARP3 interacts with and ADP-ribosylates GSK3 $\beta$, and limits the ubiquitination of Rictor}

In cancer cells, the levels of Rictor are regulated by its GSK3 $\beta$-dependent ubiquitination and proteasomal degradation [28]. Moreover, GSK3 $\beta$ has been identified in proteome wide identification of PARP3 targets [29]. To understand the biochemical basis of PARP3's contribution in Rictor/mTORC2 signaling in BRCA1-mutated cells, we investigated the association of PARP 3 with GSK3 $\beta$. We identified efficient co-immunoprecipitation of GSK3 $\beta$ with Flag-PARP3 in the PARP $3^{-l-c}$ MDA-MB436 cells (Fig. 6a). To evaluate the role of ADP-ribosylation, we measured the in vivo levels of ADP-ribosylated GSK3 $\beta$ immunoprecipitates using an anti-poly(ADP-ribose) antibody. We detected a significant basal ADP-ribosylation of GSK3 $\beta$ in the MDA-MB436 cells that was suppressed upon PARP inhibition (Fig. 6b and Supplementary Fig. 8). To further interrogate on the contribution of PARP3 in the ubiquitination of Rictor, we compared the levels of ubiquitinated Rictor immunoprecipitates in the PARP $3^{-l-c_{-}}$ MDA-MB436 versus the PARP $3^{-1-1}$-MDA-MB231 cells. Strikingly, the absence of PARP3 led to an apparent 
A

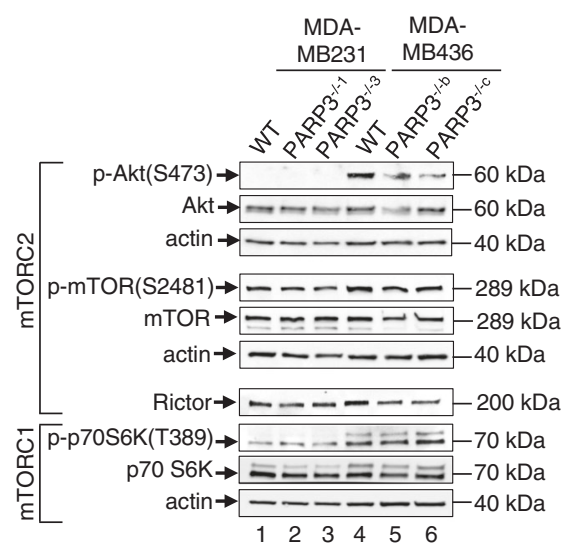

B
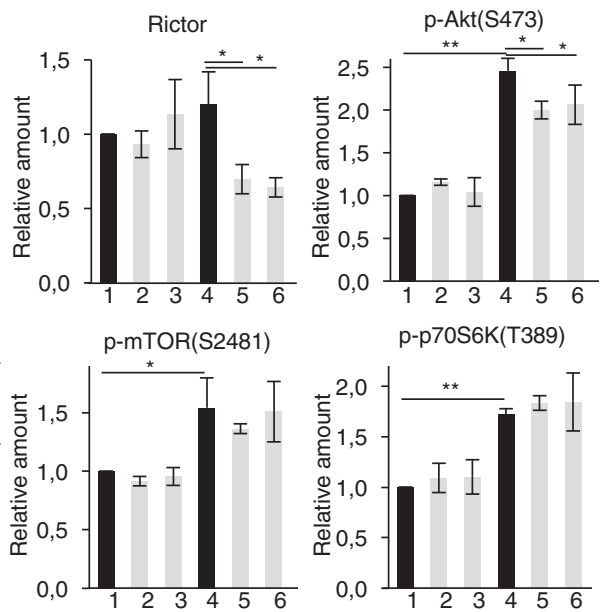
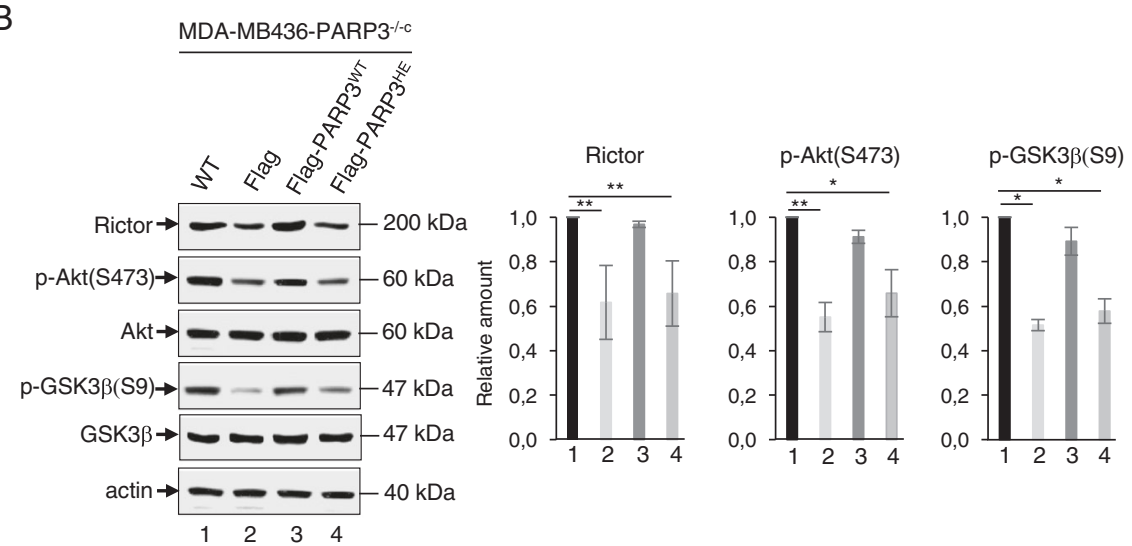

Fig. 5 The knockout of PARP3 mitigates the oncogenic mTORC2/Akt signaling pathway in BRCA1-deficient breast cancer cells. a Equivalent amounts of total cell extracts of the parental MDA-MB231 (WT) and the two PARP $3^{-l-1}$ and PARP $3^{-l-3}$ MDA-MB231 cell lines and from the wild-type (WT) MDA-MB436 cells versus the PARP3 ${ }^{-1-b}$ and PARP $3^{-l-\mathrm{c}}$ MDA-MB436 clones were immunoblotted with antibodies against p-Akt(S473) and Akt, p-mTOR(S2481) and mTOR, and Rictor as markers of mTORC2 activity and against p-p70S6K (T389) as a marker of mTORC1 activity. Actin was used as loading control (left panel). The relative signal intensities of p-Akt(S473) versus Akt, p-mTOR(S2481) versus mTOR, p-p70S6K (T389) versus p70S6K and Rictor versus actin were measured in three independent experiments using ImageJ (right panel). Mean values \pm SD are

increase in the ubiquitination levels of Rictor in the PARP3 ${ }^{-l-c}$-MDA-MB436 cells only (Fig. 6c). Together, these data suggest that in BRCA1-deficient cells, PARP3 interacts with and ADP-ribosylates GSK3 $\beta$ to restrain the ubiquitination of Rictor.

\section{Knockout of PARP3 decreases TGF $\beta$-dependent Rac1 activation and lamellipodia formation in BRCA1- deficient TNBC cells}

TGF $\beta$-induced activation of mTORC2 and phosphorylation of Akt at Ser473 promotes actin cytoskeleton reorganization indicated. $* p<0.05 ; * * p<0.01$. b Reintroduction of Flag-PARP3 ${ }^{\mathrm{WT}}$ in the PARP $3^{-l-\mathrm{c}}$ MDA-MB436 cell line restores the constitutive induction of p-Akt(S473), p-GSK3 $\beta$ (S9) and Rictor levels, but not the reintroduction of the dead Flag-PARP ${ }^{\mathrm{HE}}$ mutant. Equivalent amounts of total cell extracts of the parental MDA-MB436 (WT) and the PARP $^{-l-c}$ MDA-MB436 cell lines expressing either the Flag control (Flag), Flag-PARP3 ${ }^{\mathrm{WT}}$ or Flag-PARP $3^{\mathrm{HE}}$ were immunoblotted with antibodies against p-Akt(S473), Akt, Rictor, p-GSK3 $\beta$ (S9), GSK3 $\beta$, and actin used as loading control (left panel). The relative signal intensities of p-Akt(S473) versus Akt, p-GSK3 $\beta$ (S9) versus GSK3 $\beta$ and Rictor versus actin were measured in three independent experiments using ImageJ (right panel). Mean values \pm SD are indicated. $* p$ $<0.05 ; * * p<0.01$

and cell migration via a mechanism implicating the small Rho/Rac1-type GTPases [30, 31]. PARP3 drives TGF $\beta$ induced EMT and cell migration in breast cancer cells [14]. Therefore, we questioned on the contribution of PARP 3 in these oncogenic events in the context of BRCA1 deficiency. First, we evaluated the role of PARP3 in the TGF $\beta$-induced phosphorylation of Akt in our MDA-MB436 versus the MDA-MB231 cells (Fig. 7a). Relative to TGF $\beta$-untreated cells, both MDA-MB231 and MDA-MB436 cultures treated with TGF $\beta$ displayed increased levels of p-Akt(S473), but these inductions were less pronounced in the PARP3 knockout clones. Thus, the absence of PARP3 attenuates 
A

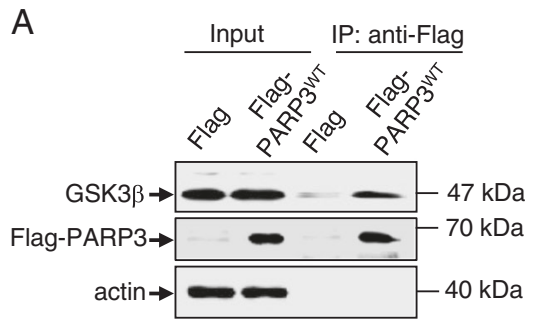

B

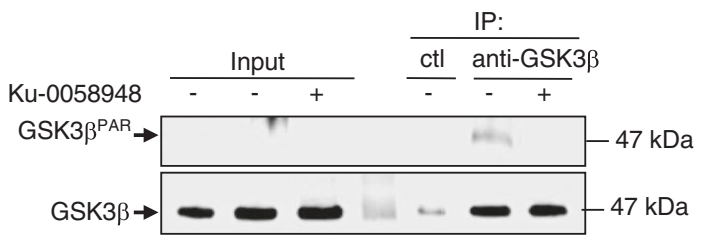

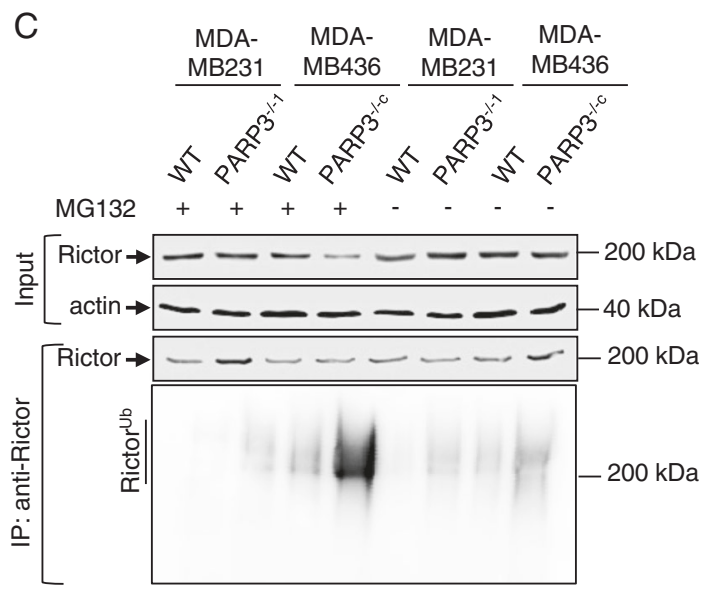

Fig. 6 PARP3 interacts with GSK3 $\beta$ and selectively restrains the ubiquitination of Rictor in BRCA1-deficient breast cancer cells. a Coimmunoprecipitation of GSK3 $\beta$ with Flag-PARP3 but not with the Flag control. Cell extracts from PARP $3^{-l-\mathrm{c}}$ MDA-MB436 cell lines expressing either the Flag control (Flag) or Flag-PARP $3^{\text {WT }}$ were immunoprecipitated with an anti-Flag antibody and analysed by western blotting using successively anti-GSK3 $\beta$, anti-Flag, and antiactin antibodies. Input corresponds to $1 / 10$ of the total amount of cell extract used for immunoprecipitation. b GSK3 $\beta$ is ADP-ribosylated in the BRCA1-mutated MDA-MB436 cells. MDA-MB436 cell extracts were immunoprecipitated with either a control antibody or an antiGSK3 $\beta$ antibody in the absence or in the presence of the PARP inhibitor Ku-0058948. ADP-ribosylated GSK3 $\beta$ was revealed using an

the TGF $\beta$-dependent activation of Rictor/mTORC2 independently of the BRCA1 context. To investigate this result further, we measured TGF $\beta$-induced RhoA and Rac1 GTPase activities in our cell models (Fig. 7b). Efficient TGF $\beta$-induced GTP loading of RhoA was detected in both MDA-MB231 and MDA-MB436 cells. A similar weak decrease was observed in both PARP3 knockout clones suggesting a faint contribution of PARP 3 in the activation of RhoA independently of the BRCA1 context. In contrast, MDA-MB436 cells displayed a notably higher TGF $\beta$ induced GTP loading of Rac1 relative to the MDA-MB231 cells suggesting a specific hyperactivation of Rac1 in BRCA1-deficient cells. This induction was absent in the two $\mathrm{PARP}^{-l-\mathrm{b}}$ and PARP3 ${ }^{-l-\mathrm{c}}$ MDA-MB436 clones. Conversely, the absence of PARP3 had no consequence on the induction of Rac1 in the MDA-MB231 cells. Hence, TGF $\beta$-induced Rac1 activity is profoundly impaired in the absence of PARP3 in the context of BRCA1 deficiency.
anti-poly(ADP-ribose) antibody and an anti-GSK3 $\beta$ antibody. Input corresponds to $1 / 10$ of the total amount of cell extract used for immunoprecipitation. c In vivo ubiquitination assay: the absence of PARP3 enhances the ubiquitination of Rictor in the BRCA1-mutated MDA-MB436 cells but not in the BRCA1-wild-type MDA-MB231 cells. The parental (WT) and the PARP ${ }^{-1-1}$ MDA-MB231 cells as well as the parental (WT) and the PARP $3^{-1-\mathrm{c}}$ MDA-MB436 cells were transfected with HA-Ubiquitin and either mock-treated or treated with $10 \mu \mathrm{M}$ MG132 for $12 \mathrm{~h}$ to inhibit proteasomal degradation. Rictor immunoprecipitates were blotted successively with an anti-HA antibody to detect ubiquitinated Rictor and an anti-Rictor antibody to detected imunopurified Rictor. The weaker detection of Rictor in line 4 is due to steric hindrance caused by the enhanced ubiquitination

Rac1 signaling promotes actin filament polymerization at the leading edge of the cancer cells forming lamellipodialike protrusions, increasing cell migration [32]. Rictor/ mTORC2 converge on Rac1 to drive cell motility [33]. Therefore, we investigated whether PARP3 regulates changes in the actin cytoskeleton by staining F-actin (Fig. 7c). TGF $\beta$ treatment resulted in the appearance of lamellipodia in the MDA-MB436 cells while no such structures were detected in the MDA-MB231 cells. In agreement with impaired Rac1 activation, the knockout of PARP3 significantly reduced the extent of lamellipodia formation in the two $\mathrm{PARP}^{-1-\mathrm{b}}$ and $\mathrm{PARP} 3^{-/-\mathrm{c}}$ MDAMB436 clones. No effect was seen in the PARP3 ${ }^{-1-1}$ and $\mathrm{PARP}^{-1-3}$ MDA-MB231 clones. We conclude that the absence of PARP3 impairs the Rac1-mediated formation of lamellipodia structures in the context of BRCA1 deficiency. Consequently, we compared the motility of our wild-type and PARP3-knockout cell models (Fig. 7d). The absence of 

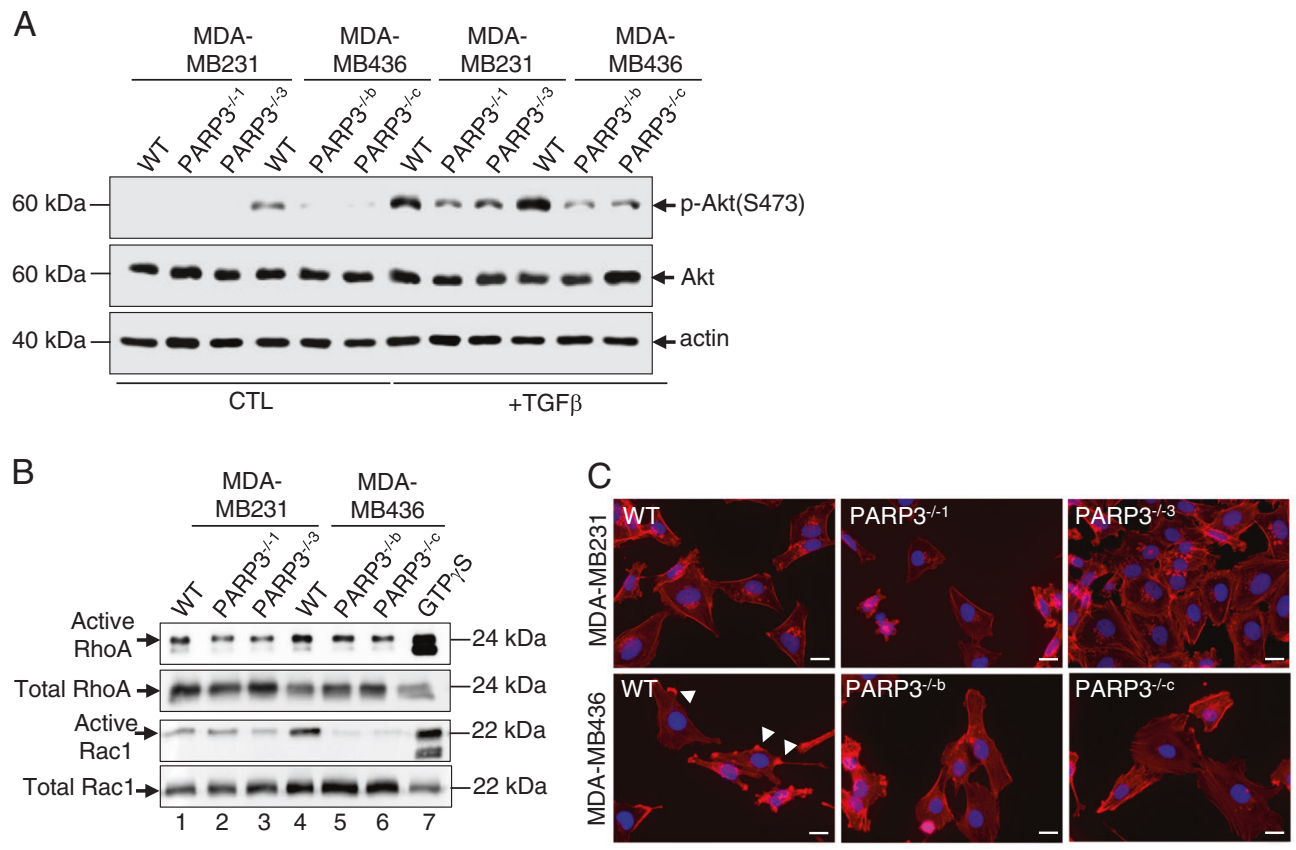

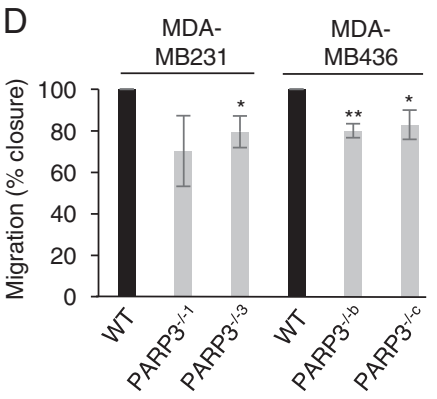

Fig. 7 The knockout of PARP3 diminishes the TGF $\beta$-dependent Rac1 activation and lamellipodia formation in BRCA1-deficient breast cancer cells. a The parental MDA-MB231 (WT) and the two PARP3 ${ }^{-1-1}$ and PARP $3^{-/-3}$ MDA-MB231 knockout cell lines and the wildtype (WT) MDA-MB436 cells versus the PARP3 $3^{-1-\mathrm{b}}$ and $\mathrm{PARP}^{-1-\mathrm{c}}$ MDA-MB436 clones were either mock-treated (CTL) or stimulated with TGF $\beta$ for $48 \mathrm{~h}$ (+TGF $\beta$ ). Equivalent amounts of total cell lysates were immunoblotted with antibodies against p-Akt(S473), Akt and actin as loading control. b The parental MDA-MB231 (WT) and the two PARP3 $3^{-1-1}$ and PARP3 $3^{-1-3}$ MDA-MB231 knockout cell lines and the wild-type (WT) MDA-MB436 cells versus the PARP ${ }^{-1-\mathrm{b}}$ and PARP $3^{-1-\mathrm{c}}$ MDA-MB436 clones were stimulated with TGF $\beta$ for $48 \mathrm{~h}$. Active GTP-bound RhoA or active GTP-bound Racl were isolated using GST-Rhotekin-RBD-Sepharose, and detected by immunoblotting using antibodies against RhoA and Rac1, respectively. Total

PARP3 decreased cell migration independently of the BRCA1 context.

\section{Chemical inhibition of PARP3 recapitulates the effects of the genetic disruption of PARP3}

To further validate the impact of PARP3 inhibition in BRCA1-deficient TNBC cells, we used the cell-permeable PARP3-specific inhibitor, ME0328 [16]. We first compared the sensitivity of the MDA-MB231 and MDA-MB436 cells abundance of RhoA and Rac1 was analysed in the input using the appropriate antibodies. $\mathbf{c}$ The parental MDA-MB231 (WT) and the two $\mathrm{PARP}^{-1-1}$ and PARP ${ }^{-1-3}$ MDA-MB231 knockout cell lines and the wild-type (WT) MDA-MB436 cells versus the PARP3 $^{-1-b}$ and PARP $3^{-l-\mathrm{c}}$ MDA-MB436 clones were stimulated with TGF $\beta$ for $48 \mathrm{~h}$ and immunostained with Alexa Fluor 647 Phalloidin (red) to examine F-actin stress fibers and lamellipodia formation indicated by white arrows. Nuclei are counterstained with DAPI (blue). d Analysis of video recording of the in vitro scratch-wounds. The percentage of wound closure was calculated by the mean \pm SD of five wound widths per condition taken at different time points throughout $24 \mathrm{~h}$ and performed in three independent experiments. The percentage of wound closure for the parental cells was set to $100 \%$. $* p<0.05 ; * * p<0.01$. Scale bars $=10 \mu \mathrm{m}$

to increasing doses of ME0328 by clonogenic assays (Fig. 8a). At all doses of ME0328 used, the inhibition of PARP3 caused significantly higher lethality in the BRCA1deficient cells then in the BRCA1-wild-type cells (black bars). Similar result was observed in the HCC1937 cells (Supplementary Fig. 7A). Notably, this effect was not detected in the PARP $3^{-1-\mathrm{c}}$ MDA-MB436 cells and not significant at the highest dose in the PARP $3^{-1-1}$ MDAMB231 cells (gray bars) revealing the specificity of ME0328 in vivo. 
A

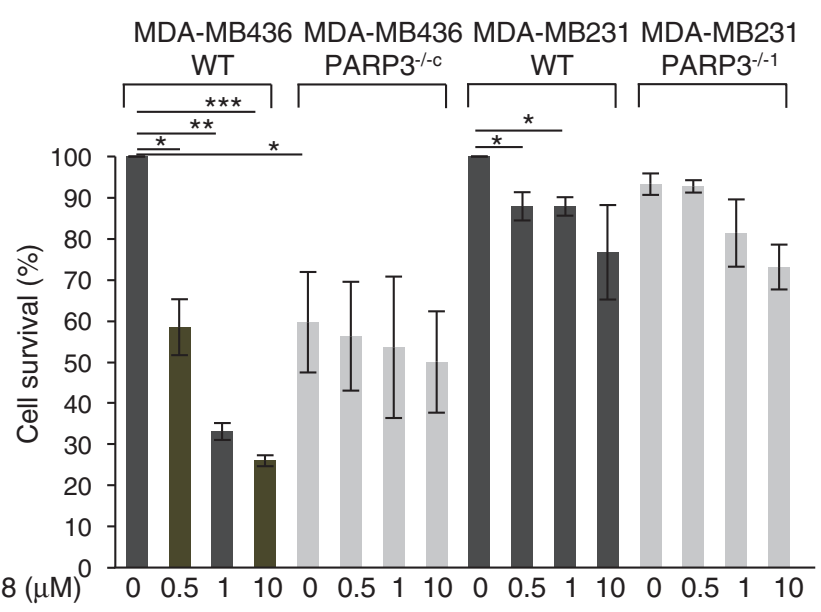

B

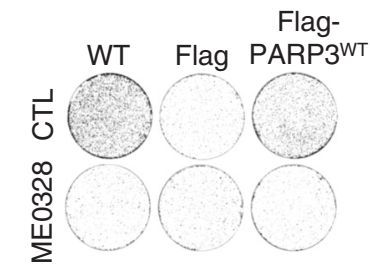

Mock-treated +ME0328, $1 \mu \mathrm{M}$

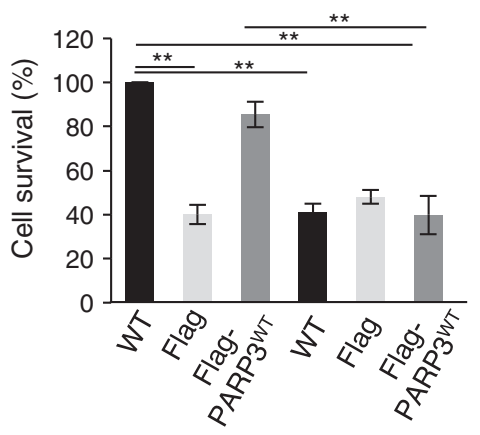

C

MDA-MB436 PARP3 $3^{-1-c}$
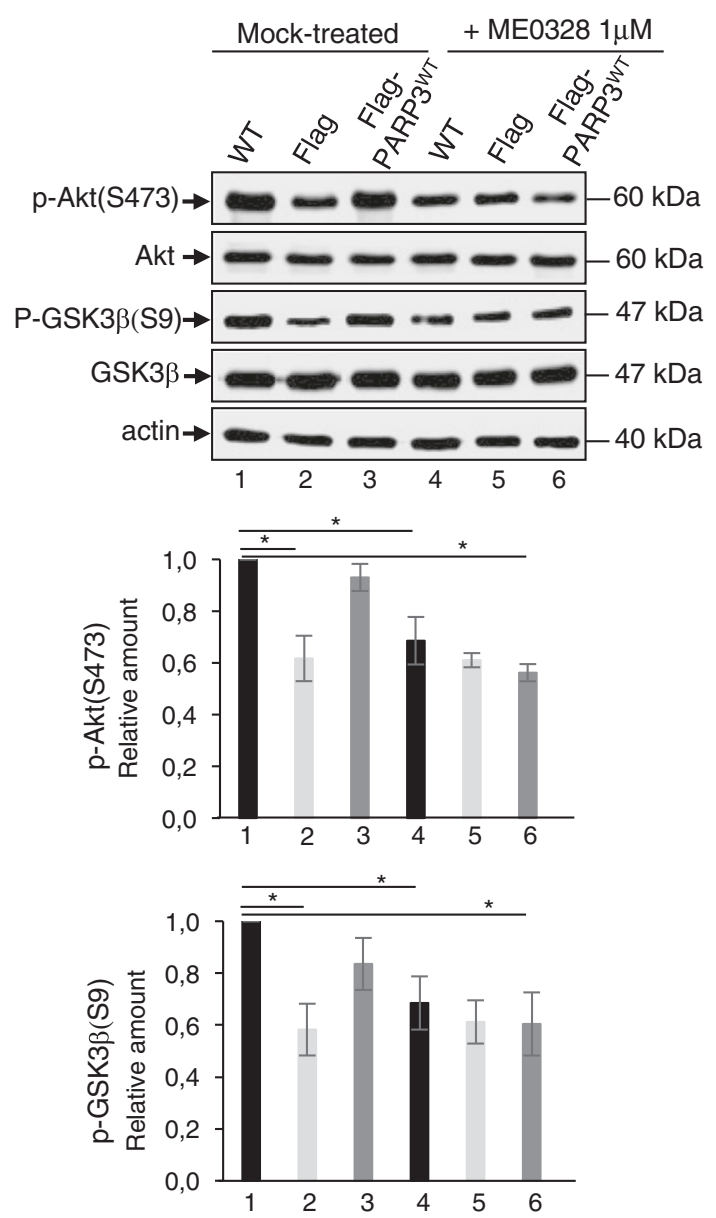

Clonogenic survival of the parental MDA-MB436 cell line was set to $100 \%$. Results represent the mean values of three independent experiments \pm SD. $* * p<0.01$. c The parental MDA-MB436 (WT) and the PARP3 ${ }^{-1-\mathrm{c}}$ MDA-MB436 cell lines expressing either the Flag control (Flag), Flag$\mathrm{PARP}^{\mathrm{WT}}$, or Flag-PARP3 ${ }^{\mathrm{HE}}$ were either mock-treated or exposed to ME0328 for $24 \mathrm{~h}$ and processed for the analysis of mTORC2 signaling by western blotting using the appropriate antibodies (upper panel). The relative signal intensities of p-Akt(S473) versus Akt, and p-GSK3ß(S9) versus GSK3 $\beta$ were measured in three independent experiments using ImageJ (right panel). Mean values \pm SD are indicated. $* p<0.05$ 
We then analysed the sensitivity of the MDA-MB436 cells to the compound compared to the PARP $3^{-l-\mathrm{c}}$ MDAMB436 cells expressing either Flag or Flag-PARP3 ${ }^{\text {WT }}$ (Fig. 8b). ME0328 significantly reduced survival of the control MDA-MB436 cells and the PARP3 ${ }^{-1-\mathrm{c}}$ MDAMB436 cells restored with Flag-PARP $3^{\text {WT }}$, but had no impact on the PARP $3^{-1-\mathrm{c}}$ MDA-MB436 cells expressing the Flag tag only, confirming the selectivity to PARP3 inhibition.

To determine the inhibitory effect of ME0328 on Rictor/ mTORC2 signaling, we analysed the status of p-Akt(S473) and p-GSK3 $\beta(\mathrm{S} 9)$ in the MDA-MB436 restored cells upon exposure to ME0328 (Fig. 8c). Compared to untreated cells, ME0328 significantly decreased the levels of p-Akt(S473) and p-GSK3 $\beta(S 9)$ in WT and PARP $3^{-1-\mathrm{c}}$ MDA-MB436 cells restored with Flag-PARP ${ }^{\mathrm{WT}}$, but had no effect on the PARP3 $^{-1-\mathrm{c}}$ MDA-MB436 expressing the Flag tag only. Similarly, ME0328 reduced the levels of p-Akt(S473) and p-GSK3 $\beta$ (S9) in HCC1937 cells (Supplementary Fig. 7C). Together, these results confirmed that the chemical inhibition of PARP3 reduces the survival and Rictor/mTORC2 activity in the context of BRCA1 deficiency specifically.

\section{Discussion}

Analysis of the published PARP3 gene expression profile using the Cancer Cell Line Encyclopedia (CCLE) shows a significantly higher expression of $P A R P 3$ in the TNBC versus the non-TNBC cells, whereas PARPI expression remains similar (Supplementary Fig. 1). TNBC have a high prevalence of BRCAl mutations [34]. It has been demonstrated previously that inhibition of Tankyrase 1 efficiently reduces survival of BRCA1-associated cancers [20]. We found that PARP3 stimulates Tankyrase 1 activity [8]. On these bases, we hypothesized that targeting PARP3 may provide a novel therapeutic avenue for BRCA1-deficient TNBC.

We demonstrate here that the knockdown and knockout of PARP3, or inhibition of its catalytic activity is selectively lethal with BRCA1 in several experimental models of TNBC. We discovered an unprecedented selective role of PARP3 in the regulation of mTORC2 complex, a critical Akt Ser473 kinase [7]. Although mTORC2 complex is gaining attention as a relevant target for cancer therapy due to its important functions in cell survival, cytoskeleton organization, cell migration, and metabolism, its contribution in the progression of the BRCA1-deficient breast cancers has not yet been evaluated [6]. Here we provide robust evidence that loss of PARP3 or inhibition of its catalytic activity attenuates mTORC2-mediated Akt phosphorylation especially in BRCA1-deficient cells that upregulate p-Akt (S473) showing a lesser effect in cells with normal BRCA1 expression. The mechanism by which PARP3-catalyzed
ADP-ribosylation positively regulates $\mathrm{mTORC} 2$ appears to be associated with the interaction of PARP3 with GSK3 $\beta$ that might be required to temper the ubiquitination and degradation of Rictor [35]. Rictor/mTORC2 pathway has been reported to be an essential downstream branch of TGF $\beta$ signaling representing a responsive target to inhibit EMT [31]. We have shown that PARP3 drives TGF $\beta$ and ROS-induced EMT, migration and stemness in breast cancer cells [14]. An important morphological modification induced by TGF $\beta$ during migration involves the rearrangement of the cytoskeleton leading to formation of lamellipodia, a phenomenon elevated in BRCA1-deficient breast cancer cells and associated with increased activation of the small GTPases, RhoA and Rac1 [32, 36]. Rictor/mTORC2 plays a role in cytoskeleton reorganization and Rac1 has been involved [35, 37, 38]. We show here that PARP3 regulates these events in a context-dependent manner. Our data suggest that PARP3 promotes TGF $\beta$-induced p-Akt (S473), RhoA activation and consequently migration irrespective of the BRCA1 context. These findings introduce signaling through RhoA as a downstream mechanism by which PARP3 may promote migration in breast cancer cells. In contrast, PARP3 supports enhanced Rac1 activation and lamellipodia formation in the context of BRCA1 deficiency reinforcing the hypothesis that targeting PARP3 could be beneficial to restrain BRCA1-associated cancer progression.

Moreover, cumulative evidences point to a central role of mTORC2 signaling in the maintenance of genome integrity and deregulated p-Akt(S473) has been linked with surnumerary centrosomes [39-41]. Accordingly, we found that the absence of PARP3 results in centrosome amplification and genomic instability as measured by the accumulation of micronuclei, telomeric aberrations and an increase in $\gamma \mathrm{H} 2 \mathrm{AX}$ and P-ATM levels that are intensified in the BRCA1-deficient cells (Supplementary Fig. 9). Hence, it is tempting to correlate abnormal centrosome numbers and genome instability with reduced Rictor/mTORC2 signaling in the absence of PARP3.

In conclusion, we identified a new role of PARP3 catalyzed ADP-ribosylation in promoting Rictor/ mTORC2 signaling and Rac1 GTPase activation specifically in BRCA1-deficient TNBC cells emphasizing the potential clinical implications of selective PARP3 inhibitors. Accordingly, we demonstrate the efficacy and selectivity of the recently reported quinazoline derivative ME0328 as this compound compromises survival and mTORC2 signaling in the wild-type MDA-MB436 cells but not in the PARP3 knockout counterparts. These data exemplify PARP3 as a prominent target in a precision medicine approach and thus support the significant drug design efforts developed since a few years for selective targeting of PARPs in cancer therapy. 
Acknowledgements This work was supported by the Association pour la Recherche contre le Cancer, Ligue Nationale Contre le Cancer, CNRS, Université de Strasbourg and Ramon Areces Foundation. This work has been published within the LABEX ANR-10-LABX-0034_Medalis.

\section{Compliance with ethical standards}

Conflict of interest The authors declare that they have no conflict of interest.

\section{References}

1. Gupte R, Liu Z, Kraus WL. PARPs and ADP-ribosylation: recent advances linking molecular functions to biological outcomes. Genes Dev. 2017;31:101-26.

2. Steffen JD, Brody JR, Armen RS, Pascal JM. Structural implications for selective targeting of PARPs. Front Oncol. 2013;3:301.

3. Turner NC, Reis-Filho JS. Basal-like breast cancer and the BRCA1 phenotype. Oncogene. 2006;25:5846-53.

4. Werfel TA, Wang S, Jackson MA, Kavanaugh TE, Morrison Joly M, Lee L, et al. Selective mTORC2 inhibitor therapeutically blocks breast cancer cell growth and survival. Cancer Res. 2018;78:1845-1858.

5. Laplante M, Sabatini DM. mTOR signaling at a glance. J Cell Sci. 2009;122(Pt 20):3589-94.

6. Oh WJ, Jacinto E. mTOR complex 2 signaling and functions. Cell Cycle. 2011;10:2305-16.

7. Sarbassov DD, Guertin DA, Ali SM, Sabatini DM. Phosphorylation and regulation of Akt/PKB by the rictor-mTOR complex. Science. 2005;307:1098-101.

8. Boehler C, Gauthier LR, Mortusewicz O, Biard DS, Saliou JM, Bresson A, et al. Poly(ADP-ribose) polymerase 3 (PARP3), a newcomer in cellular response to DNA damage and mitotic progression. Proc Natl Acad Sci USA. 2011;108: 2783-8.

9. Robert I, Gaudot L, Rogier M, Heyer V, Noll A, Dantzer F, et al. Parp3 negatively regulates immunoglobulin class switch recombination. PLoS Genet. 2015;11:e1005240.

10. Rouleau M, Saxena V, Rodrigue A, Paquet ER, Gagnon A, Hendzel MJ, et al. A key role for poly(ADP-Ribose) polymerase 3 in ectodermal specification and neural crest development. PLoS ONE. 2011;6:e15834.

11. Rulten SL, Fisher AE, Robert I, Zuma MC, Rouleau M, Ju L, et al. PARP-3 and APLF function together to accelerate nonhomologous end-joining. Mol Cell. 2011;41:33-45.

12. Grundy GJ, Polo LM, Zeng Z, Rulten SL, Hoch NC, Paomephan $\mathrm{P}$, et al. PARP3 is a sensor of nicked nucleosomes and monoribosylates histone $\mathrm{H} 2 \mathrm{~B}(\mathrm{Glu} 2)$. Nat Commun. 2016;7: 12404.

13. Day TA, Layer JV, Cleary JP, Guha S, Stevenson KE, Tivey T, et al. PARP3 is a promoter of chromosomal rearrangements and limits G4 DNA. Nat Commun. 2017;8:15110.

14. Karicheva O, Rodriguez-Vargas JM, Wadier N, MartinHernandez K, Vauchelles R, Magroun N, et al. PARP3 controls TGFbeta and ROS driven epithelial-to-mesenchymal transition and stemness by stimulating a TG2-Snail-E-cadherin axis. Oncotarget. 2016;7:64109-23.

15. Lindgren AE, Karlberg T, Ekblad T, Spjut S, Thorsell AG, Andersson CD, et al. Chemical probes to study ADP-ribosylation: synthesis and biochemical evaluation of inhibitors of the human ADP-ribosyltransferase ARTD3/PARP3. J Med Chem. 2013;56:9556-68.

16. Lindgren AE, Karlberg T, Thorsell AG, Hesse M, Spjut S, Ekblad T, et al. A PARP inhibitor with selectivity toward ADP- ribosyltransferase ARTD3/PARP3. ACS Chem Biol. 2013;8:1698-703.

17. Farmer H, McCabe N, Lord CJ, Tutt AN, Johnson DA, Richardson TB, et al. Targeting the DNA repair defect in BRCA mutant cells as a therapeutic strategy. Nature. 2005;434:917-21.

18. Chavez KJ, Garimella SV, Lipkowitz S. Triple negative breast cancer cell lines: one tool in the search for better treatment of triple negative breast cancer. Breast Dis. 2010;32:35-48.

19. De Vos M, El Ramy R, Quenet D, Wolf P, Spada F, Magroun N, et al. Poly(ADP-ribose) polymerase 1 (PARP1) associates with E3 ubiquitin-protein ligase UHRF1 and modulates UHRF1 biological functions. J Biol Chem. 2014;289:16223-38.

20. McCabe N, Cerone MA, Ohishi T, Seimiya H, Lord CJ, Ashworth A. Targeting Tankyrase 1 as a therapeutic strategy for BRCAassociated cancer. Oncogene. 2009;28:1465-70.

21. Augustin A, Spenlehauer C, Dumond H, Menissier-De Murcia J, Piel M, Schmit AC, et al. PARP-3 localizes preferentially to the daughter centriole and interferes with the G1/S cell cycle progression. J Cell Sci. 2003;116(Pt 8):1551-62.

22. Tutt A, Gabriel A, Bertwistle D, Connor F, Paterson H, Peacock J, et al. Absence of Brca2 causes genome instability by chromosome breakage and loss associated with centrosome amplification. Curr Biol. 1999;9:1107-10.

23. Ran FA, Hsu PD, Lin CY, Gootenberg JS, Konermann S, Trevino AE, et al. Double nicking by RNA-guided CRISPR Cas9 for enhanced genome editing specificity. Cell . 2013;154: 1380-9.

24. Xiang T, Jia Y, Sherris D, Li S, Wang H, Lu D, et al. Targeting the Akt/mTOR pathway in Brcal-deficient cancers. Oncogene. 2011;30:2443-50.

25. Xiang T, Ohashi A, Huang Y, Pandita TK, Ludwig T, Powell SN, et al. Negative regulation of AKT activation by BRCA1. Cancer Res. 2008;68:10040-4.

26. Copp J, Manning G, Hunter T. TORC-specific phosphorylation of mammalian target of rapamycin (mTOR): phospho-Ser2481 is a marker for intact mTOR signaling complex 2. Cancer Res. 2009;69:1821-7.

27. Beurel E, Grieco SF, Jope RS. Glycogen synthase kinase-3 (GSK3): regulation, actions, and diseases. Pharmacol Ther. 2015;148:114-31.

28. Koo J, Wu X, Mao Z, Khuri FR, Sun SY. Rictor undergoes glycogen synthase kinase 3 (GSK3)-dependent, FBXW7mediated ubiquitination and proteasomal degradation. J Biol Chem. 2015;290:14120-9.

29. Gibson BA, Zhang Y, Jiang H, Hussey KM, Shrimp JH, Lin H, et al. Chemical genetic discovery of PARP targets reveals a role for PARP-1 in transcription elongation. Science. 2016;353:45-50.

30. Ungefroren $\mathrm{H}$, Witte $\mathrm{D}$, Lehnert $\mathrm{H}$. The role of small GTPases of the Rho/Rac family in TGF-beta-induced EMT and cell motility in cancer. Dev Dyn. 2017;247:451-461.

31. Lamouille S, Connolly E, Smyth JW, Akhurst RJ, Derynck R. TGF-beta-induced activation of mTOR complex 2 drives epithelial-mesenchymal transition and cell invasion. J Cell Sci. 2012;125(Pt 5):1259-73.

32. Gulhati P, Bowen KA, Liu J, Stevens PD, Rychahou PG, Chen M, et al. mTORC1 and mTORC2 regulate EMT, motility, and metastasis of colorectal cancer via RhoA and Rac1 signaling pathways. Cancer Res. 2011;71:3246-56.

33. Morrison Joly M, Williams MM, Hicks DJ, Jones B, Sanchez V, Young CD, et al. Two distinct mTORC2-dependent pathways converge on Rac1 to drive breast cancer metastasis. Breast Cancer Res. 2017;19:74

34. Wong-Brown MW, Meldrum CJ, Carpenter JE, Clarke CL, Narod SA, Jakubowska A, et al. Prevalence of BRCA1 and BRCA2 germline mutations in patients with triple-negative breast cancer. Breast Cancer Res Treat. 2015;150:71-80. 
35. Sarbassov DD, Ali SM, Kim DH, Guertin DA, Latek RR, Erdjument-Bromage $\mathrm{H}$, et al. Rictor, a novel binding partner of mTOR, defines a rapamycin-insensitive and raptor-independent pathway that regulates the cytoskeleton. Curr Biol. 2004;14:1296302.

36. Zhang Z, Yang M, Chen R, Su W, Li P, Chen S, et al. IBP regulates epithelial-to-mesenchymal transition and the motility of breast cancer cells via Rac1, RhoA and Cdc42 signaling pathways. Oncogene. 2014;33:3374-82.

37. Jacinto E, Loewith R, Schmidt A, Lin S, Ruegg MA, Hall A, et al. Mammalian TOR complex 2 controls the actin cytoskeleton and is rapamycin insensitive. Nat Cell Biol. 2004;6:1122-8.
38. Saci A, Cantley LC, Carpenter CL. Rac1 regulates the activity of mTORC1 and mTORC2 and controls cellular size. Mol Cell. 2011;42:50-61.

39. Weisman R, Cohen A, Gasser SM. TORC2-a new player in genome stability. EMBO Mol Med. 2014;6:995-1002.

40. Shimada K, Filipuzzi I, Stahl M, Helliwell SB, Studer C, Hoepfner $\mathrm{D}$, et al. TORC2 signaling pathway guarantees genome stability in the face of DNA strand breaks. Mol Cell. 2013;51:829-39.

41. Guan Z, Wang XR, Zhu XF, Huang XF, Xu J, Wang LH, et al. Aurora-A, a negative prognostic marker, increases migration and decreases radiosensitivity in cancer cells. Cancer Res. 2007;67:10436-44. 